\title{
Synthesis of Graft Polymer*1 with Controlled Stereoregularities in the Main Chain and in the Side Chains by Stereospecific Copolymerization of Isotactic Poly(methyl methacrylate) Macromonomer with Methacrylate Monomer
}

\author{
Tatsuki Kitayama, Osamu Nakagawa, and Koichi Hatada \\ Department of Chemistry, Faculty of Engineering Science, \\ Osaka University, Toyonaka, Osaka 560, Japan
}

(Received May 11, 1995)

\begin{abstract}
Highly isotactic poly(methyl methacrylate) (PMMA) macromonomer having methacryloyl group as a polymerizable function was synthesized from PMMA living anion prepared with $t-\mathrm{C}_{4} \mathrm{H}_{9} \mathrm{MgBr}$ in toluene at $-78^{\circ} \mathrm{C}$. Anionic copolymerizations of the PMMA macromonomer with methyl or ethyl methacrylate were carried out to obtain stereoregular graft polymers. ${ }^{* 1}$ Copolymerizations by 1,1 -diphenylhexyllithium in tetrahydrofuran at $-78^{\circ} \mathrm{C}$ and by $t-\mathrm{C}_{4} \mathrm{H}_{9} \mathrm{MgBr}$ in toluene at $-78^{\circ} \mathrm{C}$ gave graft polymers with syndiotactic and isotactic main chains, respectively, as confirmed by ${ }^{13} \mathrm{C}$ NMR spectroscopy. Some of solution properties of the stereoregular graft polymers were investigated. The graft polymers with isotactic and syndiotactic PMMA chains were found to exhibit peculiar viscosity behavior due to stereocomplex formation.
\end{abstract}

KEY WORDS Macromonomer/Graft Polymer / Stereoregularity / Anionic

Copolymerization / Methacrylate Monomer / Poly(methyl methacrylate) /

NMR / End Group / Stereocomplex /

Recent advances of living polymerization have made it possible to prepare polymers with controlled structure, such as end-functional polymer, block polymer, graft polymer, starlike polymer and so on. Among them, macromonomer is one of the most attractive endfunctional polymers derived from living polymers, and has been proved to provide an effective way for the structural control of branched polymers such as graft polymers and comblike polymers. ${ }^{1-13}$ Utilizing our findings of stereospecific living polymerizations of methacrylate ${ }^{14,15}$ we have prepared stereoregular poly(methyl methacrylate) (PMMA) macromonomers having styrene-type group ${ }^{6-10,13}$; polymerization and copolymerization of these macromonomers could be used without doubt to control the stereoregularity of side chains in comblike and graft polymers. Recently, we reported the synthesis of syndiotactic (st-) PMMA macromonomer with methacryloyl group, anionic copolymerizations of which with low-molecular-weight methacrylate monomers afforded graft polymers with controlled stereoregularities both in the main chain and in the side chains. ${ }^{12,13}$ The reason why methacryloyl group was selected as a polymerizable function is that methacrylate is one of the few monomers whose stereospecific polymerizations have been well surveyed. ${ }^{16,17}$ In the present work, an isotactic (it-) PMMA macromonomer with the same chemical structure as the $s t$-macromonomer was prepared and copolymerized anionically with methyl (MMA) or ethyl (EMA) methacrylate to obtain stereoregular graft polymers with

*1 In this paper, "graft polymer" is used to describe the products from copolymerization of macromonomer and comonomer, although some can be termed graft copolymer and some branched polymer. See ref 12 . 
$i t$-PMMA side chains. Some of solution properties of the stereoregular graft polymers with it-PMMA side chains were investigated and compared with those of graft polymer with $s t$-PMMA side chains reported in the previous papers. $^{12,13}$

\section{EXPERIMENTAL}

\section{Materials}

Monomers and solvents used were purified by the same manners described in the previous paper. ${ }^{12}$ 1,8-Diazabicyclo[5.4.0]undec-7-ene (DBU) was purified by distillation. The details for other reagents were described in the previous paper. ${ }^{12}$

\section{Preparation of it-PMMA Macromonomer}

Isotactic PMMA macromonomer having methacryloyl function was prepared from it-PMMA anion as shown in Scheme 1. The procedure for the preparation of it-PMMA macromonomer is almost the same as that of $s t$-macromonomer. ${ }^{12}$

To it-PMMA living anions formed with $t-\mathrm{C}_{4} \mathrm{H}_{9} \mathrm{MgBr}$ in toluene at $-78^{\circ} \mathrm{C},{ }^{14} 10$ equivalents of allyl iodide were added slowly at $-78^{\circ} \mathrm{C}$ to obtain $i t$-PMMA with allyl end group (it-PMMA-CH${ }_{2} \mathrm{CH}=\mathrm{CH}_{2}$ ). After $1 \mathrm{~h}, 5$ equivalents of $\mathrm{DBU}$ in toluene were added to this mixture, and the reaction was continued for $24 \mathrm{~h}$ at $-78^{\circ} \mathrm{C}$ and then for further $3 \mathrm{~h}$ at $0^{\circ} \mathrm{C}$. The reaction mixture was poured into a large amount of hexane. The precipitated polymer was collected by filtration, washed successively with hexane, diluted aqueous $\mathrm{HCl}$ and water, and dried under vacuum at room temperature. The polymer was redissolved in benzene and recovered by freeze-drying after removing undissolved materials by centrifugation.

The it-PMMA macromonomer was synthesized by the reaction between it-PMMA having hydroxyl end group (it-PMMA- $\mathrm{CH}_{2} \mathrm{CH}_{2}$ $\mathrm{CH}_{2} \mathrm{OH}$ ), which was derived from the it -PMMA- $\mathrm{CH}_{2} \mathrm{CH}=\mathrm{CH}_{2}$ through hydrobo- ration with 9-borabicyclo[3.3.1] nonane (9-BBN), and methacryloyl chloride in the presence of pyridine (Scheme 1). The procedures for the syntheses of $i t-\mathrm{PMMA}-\mathrm{CH}_{2} \mathrm{CH}_{2}$ $\mathrm{CH}_{2} \mathrm{OH}$ and it-PMMA macromonomer were similar to those of $s t$-one as described in the previous paper. ${ }^{12}$

\section{Anionic Copolymerization of it-PMMA Macro- monomer}

The detailed procedures for copolymerizations of PMMA macromonomer with MMA or EMA were described in the previous paper. ${ }^{12}$ The copolymerizations of it-PMMA macromonomer were carried out in glass ampoules filled with dry nitrogen. After a certain polymerization time, a small amount of methanol containing $\mathrm{HCl}\left(1 \mathrm{moll}^{-1}\right)$ was added to the polymerization mixture to quench the polymerization reaction. The solvent was removed under vacuum, the residue was dissolved in benzene and centrifuged to remove insoluble materials, and then the product mixture was recovered by freeze-drying.

The graft polymer was separated from unreacted it-PMMA macromonomer and it-PMMA without methacryloyl function by repeated reprecipitation in toluene-hexane or in chloroform-hexane mixtures.

\section{Measurements}

${ }^{1} \mathrm{H}$ NMR spectra of the polymers were recorded on a JNM GSX270 NMR spectrometer (JEOL) at $270 \mathrm{MHz} .{ }^{13} \mathrm{C}$ NMR spectra of the polymers were measured on the JNM GSX270 NMR spectrometer at $67.8 \mathrm{MHz}$.

Gel permeation chromatography (GPC) was performed on a JASCO 880-PU chromatograph equipped with Shodex GPC columns $\mathrm{KF}-80 \mathrm{M}(30 \mathrm{~cm} \times 0.80 \mathrm{~cm})$ and KF-802.5 (30 $\mathrm{cm} \times 0.80 \mathrm{~cm}$ ) with maximum porosities of $2 \times 10^{7}$ and $2 \times 10^{4}$, respectively, using tetrahydrofuran (THF) as an eluent at $40^{\circ} \mathrm{C}$. The chromatograms were calibrated with standard polystyrene samples, without taking the branching factor into account as first approx- 
imation. GPC-low angle laser light scattering (GPC-LALLS) experiments were run on the GPC system equipped with a TOSOH LS-8000 light scattering photometer as a detector.

Differential scanning calorimetric (DSC) analysis was performed on a Rigaku DSC 8230 calorimeter at a heating rate of $10^{\circ} \mathrm{C} \mathrm{min}^{-1}$.

Solution viscosities of the polymers were measured in chloroform and in acetone at $35^{\circ} \mathrm{C}$ using an Ubbelohde type viscometer.

\section{RESULTS AND DISCUSSION}

\section{Preparation of it-PMMA Macromonomer}

The it-PMMA macromonomer with methacryloyl function was synthesized from it-PMMA living anion, which was prepared with $t-\mathrm{C}_{4} \mathrm{H}_{9} \mathrm{MgBr}$ in toluene, ${ }^{14}$ by three-step reactions shown in Scheme 1. The results are summarized in Tables I-III.

The living polymerization of MMA with $t-\mathrm{C}_{4} \mathrm{H}_{9} \mathrm{MgBr}$ in toluene has been utilized in order to obtain highly isotactic PMMA macromonomer with styrene-type end group. ${ }^{8}$ From the experience in the synthesis of it-PMMA macromonomer with styrene-type end group, DBU was used to facilitate the coupling reaction between the it-PMMA living anion and allyl iodide as described in the Experimental section. The result is summarized in Table I. The functionality of allyl end group was determined as 0.95 from the ${ }^{1} \mathrm{H}$ NMR spectroscopic analysis for the $t-\mathrm{C}_{4} \mathrm{H}_{9}$ and olefinic proton signals of $i t$-PMMA- $\mathrm{CH}_{2} \mathrm{CH}=$ $\mathrm{CH}_{2}$. Number-average molecular weight $\left(\bar{M}_{n}\right)$ could be determined from the ${ }^{1} \mathrm{H}$ NMR spectra by comparing signal intensities of $\mathrm{OCH}_{3}$ and $t-\mathrm{C}_{4} \mathrm{H}_{9}$ protons.

The results of the syntheses of it-PMMA$\mathrm{CH}_{2} \mathrm{CH}_{2} \mathrm{CH}_{2} \mathrm{OH}$ and it-PMMA macromonomer are shown in Tables II and III, respectively. The values of functionality and $\bar{M}_{n}$ of these two it-polymers were determined by the ${ }^{1} \mathrm{H}$ NMR spectroscopic analysis in a similar manner to that for the corresponding stpolymers described in the previous papers. ${ }^{12,13}$ The it-PMMA macromonomer with methacryloyl function having the same chemical structure as the st-PMMA macromonomer was obtained with a good functionality.

\section{Anionic Copolymerization of it-PMMA Macro- monomer}

Anionic copolymerizations of the it-PMMA macromonomer with MMA or EMA were carried out in toluene with $t-\mathrm{C}_{4} \mathrm{H}_{9} \mathrm{MgBr}$ and in THF by 1,1-diphenylhexyllithium (DPHLi)
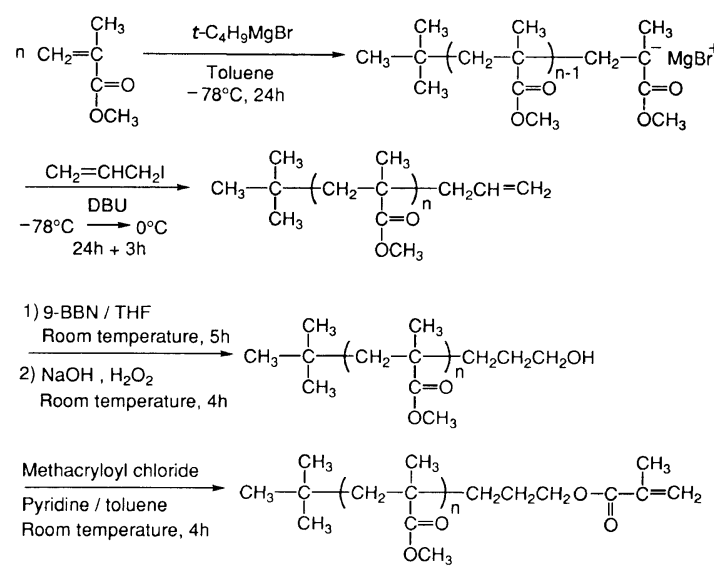

Scheme 1. Preparation of it-PMMA macromonomer.

Table I. Preparation of it-PMMA with allyl end group

\begin{tabular}{|c|c|c|c|c|c|c|c|c|c|c|}
\hline \multirow{2}{*}{$\frac{\text { MMA }}{\mathrm{mmol}}$} & \multirow{2}{*}{$\frac{t-\mathrm{C}_{4} \mathrm{H}_{9} \mathrm{MgBr}}{\mathrm{mmol}}$} & \multirow{2}{*}{$\frac{\text { Iodide }^{\mathrm{b}}}{\mathrm{mmol}}$} & \multirow{2}{*}{$\frac{\mathrm{DBU}^{\mathrm{c}}}{\mathrm{mmol}}$} & \multirow{2}{*}{$\begin{array}{c}\text { Yield } \\
\%\end{array}$} & \multirow{2}{*}{$\bar{M}_{n}^{\mathrm{d}}$} & \multirow{2}{*}{$\frac{\bar{M}_{w}^{\mathrm{e}}}{\bar{M}_{n}}$} & \multicolumn{3}{|c|}{ Tacticity $^{d}(\%)$} & \multirow{2}{*}{$\begin{array}{c}f^{\mathrm{f}} \\
\%\end{array}$} \\
\hline & & & & & & & $m m$ & $m r$ & $r r$ & \\
\hline 103 & 4.0 & 42.0 & 21.0 & 94 & 2910 & 1.11 & 94 & 3 & 3 & 95 \\
\hline
\end{tabular}

${ }^{a}$ Polymerization was carried out in toluene at $-78^{\circ} \mathrm{C}$ for $24 \mathrm{~h} .{ }^{\mathrm{b}}$ Allyl iodide. ${ }^{\mathrm{c}}$ Added as a toluene solution. ${ }^{\mathrm{d}}$ Determined by ${ }^{1} \mathrm{H}$ NMR spectroscopy. ${ }^{\mathrm{e}}$ Determined by GPC. ${ }^{\mathrm{f}}$ Functionality of allyl group determined by ${ }^{1} \mathrm{H}$ NMR spectroscopy. 
Table II. Preparation of it-PMMA with hydroxyl end group

\begin{tabular}{lc}
\hline THF & $97 \mathrm{ml}$ \\
it $-\mathrm{PMMA}-\mathrm{CH}_{2}-\mathrm{CH}=\mathrm{CH}_{2}{ }^{\mathrm{a}}$ & $9.71 \mathrm{~g}$ \\
$\mathrm{Allyl}$ end group & $3.17 \mathrm{mmol}$ \\
$9-\mathrm{BBN}^{\mathrm{b}}$ & $31.7 \mathrm{mmol}$ \\
$\mathrm{NaOH}^{\mathrm{c}}$ & $32 \mathrm{mmol}$ \\
$\mathrm{H}_{2} \mathrm{O}_{2}{ }^{\mathrm{d}}$ & $96 \mathrm{mmol}$ \\
\hline $\begin{array}{l}\text { Conversion of } \\
\quad \text { allyl end group }\end{array}$ & $95 \%$ \\
$\begin{array}{l}\text { Functionality of } \\
\quad \text { hydroxyl end group }\end{array}$ & \\
$\bar{M}_{n}{ }^{\mathrm{e}}$ (DP) & $90 \%$
\end{tabular}

${ }^{\mathrm{a}} \bar{M}_{n}=2910 \quad(\mathrm{DP}=28.1) ;$ functionality $=0.95 . \quad{ }^{\mathrm{b}} 0.5 \mathrm{M}$ THF solution. ${ }^{\mathrm{c}} 3 \mathrm{~N}$ aqueous solution. ${ }^{\mathrm{d}} 30 \%$ aqueous solution. ${ }^{\mathrm{e}}$ Determined by ${ }^{1} \mathrm{H}$ NMR spectroscopy.

Table III. Preparation of $i t$-PMMA macromonomer

\begin{tabular}{|c|c|}
\hline Toluene & $45 \mathrm{ml}$ \\
\hline$i t-\mathrm{PMMA}-\mathrm{CH}_{2} \mathrm{CH}_{2} \mathrm{CH}_{2}-\mathrm{OH}^{\mathrm{a}}$ & $4.55 \mathrm{~g}$ \\
\hline Hydroxyl end group & $1.33 \mathrm{mmol}$ \\
\hline Pyridine & $14.1 \mathrm{mmol}$ \\
\hline Methacryloyl chloride & $7.0 \mathrm{mmol}$ \\
\hline $\begin{array}{l}\text { Conversion of } \\
\text { hydroxyl end group }\end{array}$ & $99 \%$ \\
\hline $\begin{array}{l}\text { Functionality of } \\
\text { methacryloyl end group }\end{array}$ & $89 \%$ \\
\hline $\bar{M}_{n}^{\mathrm{b}}(\mathrm{DP})$ & $3140(29.5)$ \\
\hline
\end{tabular}

${ }^{\mathrm{a}} \bar{M}_{n}=3070 \quad(\mathrm{DP}=29.5)$; functionality $=0.90 .{ }^{\mathrm{b}}$ Determined by ${ }^{1} \mathrm{H}$ NMR spectroscopy. at $-78^{\circ} \mathrm{C}$. The results are shown in Table IV. The conversions of methacrylate comonomers were determined from the yield of the polymerization mixture, and found to be $100 \%$ in all the cases. The conversion of the macromonomer was determined from the ${ }^{1} \mathrm{H}$ NMR spectroscopic analysis of the polymerization mixture for the vinylidene proton signals of the methacryloyl group. The NMR analyses indicated that the conversions in the polymerization with $t-\mathrm{C}_{4} \mathrm{H}_{9} \mathrm{MgBr}$ in toluene did not reach $100 \%$, while those in the polymerization with DPHLi in THF were $100 \%$. These results mean that the rate of the polymerization at least with respect to the macromonomer was smaller for the copolymerization with $t-\mathrm{C}_{4} \mathrm{H}_{9} \mathrm{MgBr}$ in toluene than for that with DPHLi in THF, as observed for polymerizations of low-molecular-weight methacrylate. Such phenomena were observed in the copolymerization of st-PMMA macromonomer with MMA or EMA. ${ }^{12}$

In the copolymerization with MMA by $t-\mathrm{C}_{4} \mathrm{H}_{9} \mathrm{MgBr}$ in toluene, the conversion of it-PMMA macromonomer was $89 \%$ (polymerization time: 14 days) and that of $s t$-PMMA macromonomer was $65 \%$ (polymerization time: 16 days) ${ }^{12}$ while the conversions of MMA were $100 \%$ in both cases. We have reported that it-PMMA macromonomer with styrenetype end group showed higher reactivity than the corresponding st-PMMA macromonomer in radical polymerization and radical copolym-

Table IV. Anionic copolymerization of it-PMMA macromonomer (Mac.) with $t-\mathrm{C}_{4} \mathrm{H}_{9} \mathrm{MgBr}$ in toluene $[\mathrm{A}]$ and with DPHLi in THF $[\mathrm{B}]$ at $-78^{\circ} \mathrm{C}$ for 14 days

\begin{tabular}{|c|c|c|c|c|c|c|}
\hline \multirow{2}{*}{$\begin{array}{l}\text { Polymn } \\
\text { condition }\end{array}$} & \multirow{2}{*}{$\frac{\text { Mac. }}{\mathrm{mmol}}$} & \multirow{2}{*}{$\begin{array}{l}\text { Comonomer } \\
(\mathrm{mmol})\end{array}$} & \multirow{2}{*}{$\frac{\text { RMA }}{\text { Mac. }}$} & \multirow{2}{*}{$\frac{\text { Initiator }}{\mathrm{mmol}}$} & \multicolumn{2}{|c|}{ Conversion $/ \%$} \\
\hline & & & & & Mac. $^{a}$ & $\mathrm{RMA}^{\mathrm{b}}$ \\
\hline$[\mathrm{A}]$ & $0.15^{\mathrm{c}}$ & MMA (1.50) & 9.90 & 0.083 & 89 & 100 \\
\hline [A] & $0.12^{\mathrm{c}}$ & EMA (1.14) & 9.40 & 0.063 & 83 & 100 \\
\hline$[\mathrm{B}]$ & $0.12^{\mathrm{d}}$ & MMA (1.20) & 10.0 & 0.066 & 100 & 100 \\
\hline [B] & $0.11^{\mathrm{d}}$ & EMA (1.23) & 11.2 & 0.061 & 100 & 100 \\
\hline
\end{tabular}

${ }^{\mathrm{a}}$ Determined by ${ }^{1} \mathrm{H}$ NMR spectroscopy. ${ }^{\mathrm{b}}$ Determined from the yield. ${ }^{\mathrm{c}} \bar{M}_{n}=3000, \mathrm{DP}=28.1, m m: m r: r r=94: 3: 3$, $f=0.84,[\mathrm{Mac} .]_{0}=0.036\left(\mathrm{moll}^{-1}\right) .{ }^{\mathrm{d}} \bar{M}_{n}=3140, \mathrm{DP}=29.5, m m: m r: r r=94: 3: 3, f=0.89,[\mathrm{Mac} .]_{0}=0.035\left(\mathrm{moll}^{-1}\right)$. 


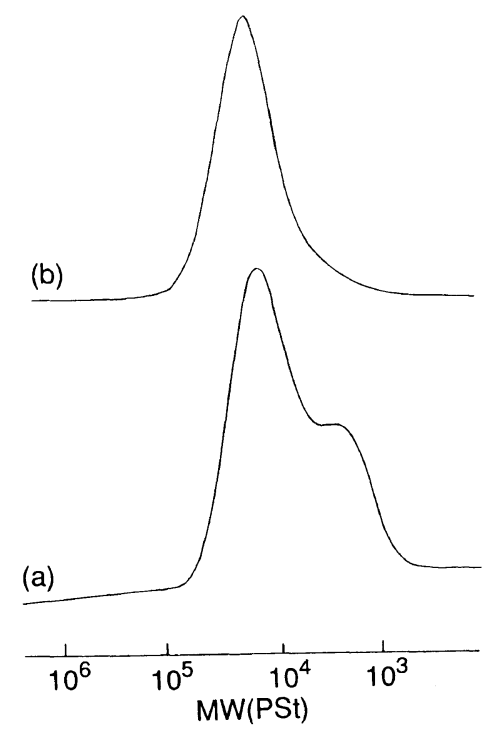

Figure 1. GPC chromatograms of the reaction mixture of the copolymerization of $i$-PMMA macromonomer with EMA by DPHLi in THF at $-78^{\circ} \mathrm{C}$ (a) and the graft polymer fractionated therefrom (b).

erization with styrene, ${ }^{8,10,13}$ and in Ziegler polymerization. ${ }^{13}$ The higher reactivity of the $i t$-macromonomer was ascribed to the higher segmental mobility of it-PMMA chain. ${ }^{18}$ The present result for the methacrylate-type PMMA macromonomers confirmed the higher reactivity of it-PMMA macromonomer than st-PMMA macromonomer, irrespective of the type of polymerizable function.

Figure la shows a GPC chromatogram for the polymerization mixture of the copolymerization of it-PMMA macromonomer and EMA with DPHLi in THF as an example. The GPC curve shows a shoulder, indicating that the polymerization mixture contained a lowmolecular-weight fraction besides a graft polymer. Since the conversion of the macromonomer reached $100 \%$ as mentioned above, the low-molecular-weight fraction should not be the starting macromonomer. Therefore, the fraction should be PMMA without methacryloyl function and the product from the macromonomer reacted but failed to propagate further. The graft polymer was isolated from the mixture by repeated precipitation in toluene-hexane mixture. The GPC curve of the graft polymer thus fractionated is shown in Figure $1 b$.

Other graft polymers except for that prepared with MMA by DPHLi in THF were isolated successfully by the same procedure. For the fractionation of the graft polymer prepared with MMA in THF, which was expected to comprise st-PMMA main chain and it-PMMA side chains as described later, chloroform-hexane mixture was used for repeated precipitation since stereocomplex formation between it- and st-PMMA segments ${ }^{19}$ may occur in toluene but not in chloroform. The stereocomplex formation of graft polymers with it-and st-PMMA segments obtained in the present work is discussed in the last section of this paper.

\section{Characterization of the Resulting Graft Polymer}

The graft polymers thus fractionated were characterized in detail by ${ }^{1} \mathrm{H}$ and ${ }^{13} \mathrm{C}$ NMR spectroscopy. The results are shown in Table $\mathrm{V}$.

Figure 2 shows ${ }^{1} \mathrm{H}$ NMR spectra of the graft polymers prepared with $t-\mathrm{C}_{4} \mathrm{H}_{9} \mathrm{MgBr}$ in toluene, together with that of the it-PMMA macromonomer. The spectrum of the it-macromonomer showed vinylidene proton signals due to the methacryloyl unit at 5.40 and $5.99 \mathrm{ppm}$ (Figure 2a). The spectra of the graft polymers (Figures $2 b$ and $2 c$ ) did not show these peaks, indicating that the graft polymers isolated from the polymerization mixtures did not contain the starting macromonomer.

Qualitative Determination of Main-Chain Tacticity In the spectrum of the graft polymer with MMA main chain (Figure 2b), the signal patterns in the regions of $\alpha-\mathrm{CH}_{3}$ and $\mathrm{CH}_{2}$ protons are almost the same as those of the it-PMMA macromonomer (Figure 2a), indicating that the main-chain tacticity is isotactic. The spectral patterns for the graft polymer with EMA main chain (Figure 2c) are more complicated, since the signals due to $\alpha-\mathrm{CH}_{3}$ and $\mathrm{CH}_{2}$ protons of EMA units show different chemical shifts from those of MMA units in 
Table V. Characteristics of graft polymers of $i t$-PMMA macromonomer (Mac.) and methacrylate comonomer (RMA) prepared with $t-\mathrm{C}_{4} \mathrm{H}_{9} \mathrm{MgBr}$ in toluene [A] and with DPHLi in THF [B]

\begin{tabular}{|c|c|c|c|c|c|c|c|c|c|c|c|c|c|c|c|}
\hline \multirow{2}{*}{$\begin{array}{l}\text { Polymn } \\
\text { condition }\end{array}$} & \multirow{2}{*}{ RMA } & \multirow{2}{*}{$\frac{\mathrm{RMA}^{\mathrm{a}}}{\text { Mac. }}$} & \multicolumn{3}{|c|}{$\bar{M}_{n}$} & \multirow{2}{*}{$\frac{\bar{M}_{w}{ }^{\mathrm{b}}}{\bar{M}_{n}}$} & \multirow{2}{*}{$N_{\mathrm{br}}^{\mathrm{c}}$} & \multicolumn{4}{|c|}{ Main chain ${ }^{\mathrm{d}}$} & \multicolumn{4}{|c|}{ Side chain ${ }^{f}$} \\
\hline & & & GPC & NMR & Calcd & & & $\mathrm{DP}^{\mathrm{e}}$ & $m m$ & $m r$ & $r r$ & $\mathrm{DP}^{\mathrm{c}}$ & $m m$ & $m r$ & $r r$ \\
\hline$[\mathrm{A}]$ & MMA & 13.1 & 15500 & 23600 & 6690 & 1.69 & 5.47 & 77.1 & 100 & 0 & 0 & 28.1 & 94 & 3 & 3 \\
\hline [A] & EMA & 8.90 & 32700 & - & 6860 & 4.37 & $8.13^{b}$ & 80.6 & 92 & 8 & 0 & 28.1 & 94 & 3 & 3 \\
\hline$[\mathrm{B}]$ & MMA & 9.41 & 9180 & 12000 & 7770 & 1.30 & 2.89 & 30.1 & 3 & 19 & 78 & 29.5 & 94 & 3 & 3 \\
\hline [B] & EMA & 7.70 & 11700 & 14600 & 8200 & 1.69 & 3.57 & 77.1 & 6 & 23 & 71 & 29.5 & 94 & 3 & 3 \\
\hline
\end{tabular}

${ }^{a}$ Composition of graft polymer. ${ }^{b}$ Determined by GPC. ${ }^{c}$ Determined by ${ }^{1} \mathrm{H}$ NMR spectroscopy. ${ }^{\mathrm{d}}$ Main-chain tacticity determined by ${ }^{13} \mathrm{C}$ NMR spectroscopy. ${ }^{\mathrm{e}} \mathrm{DP}$ (main chain) $=N_{\mathrm{br}} \times(\mathrm{RMA} / \mathrm{Mac}$. $)+N_{\mathrm{br}} \cdot{ }^{\mathrm{f}}$ Side-chain tacticity is that of the $i t$-macromonomer determined by ${ }^{1} \mathrm{H}$ NMR spectroscopy.

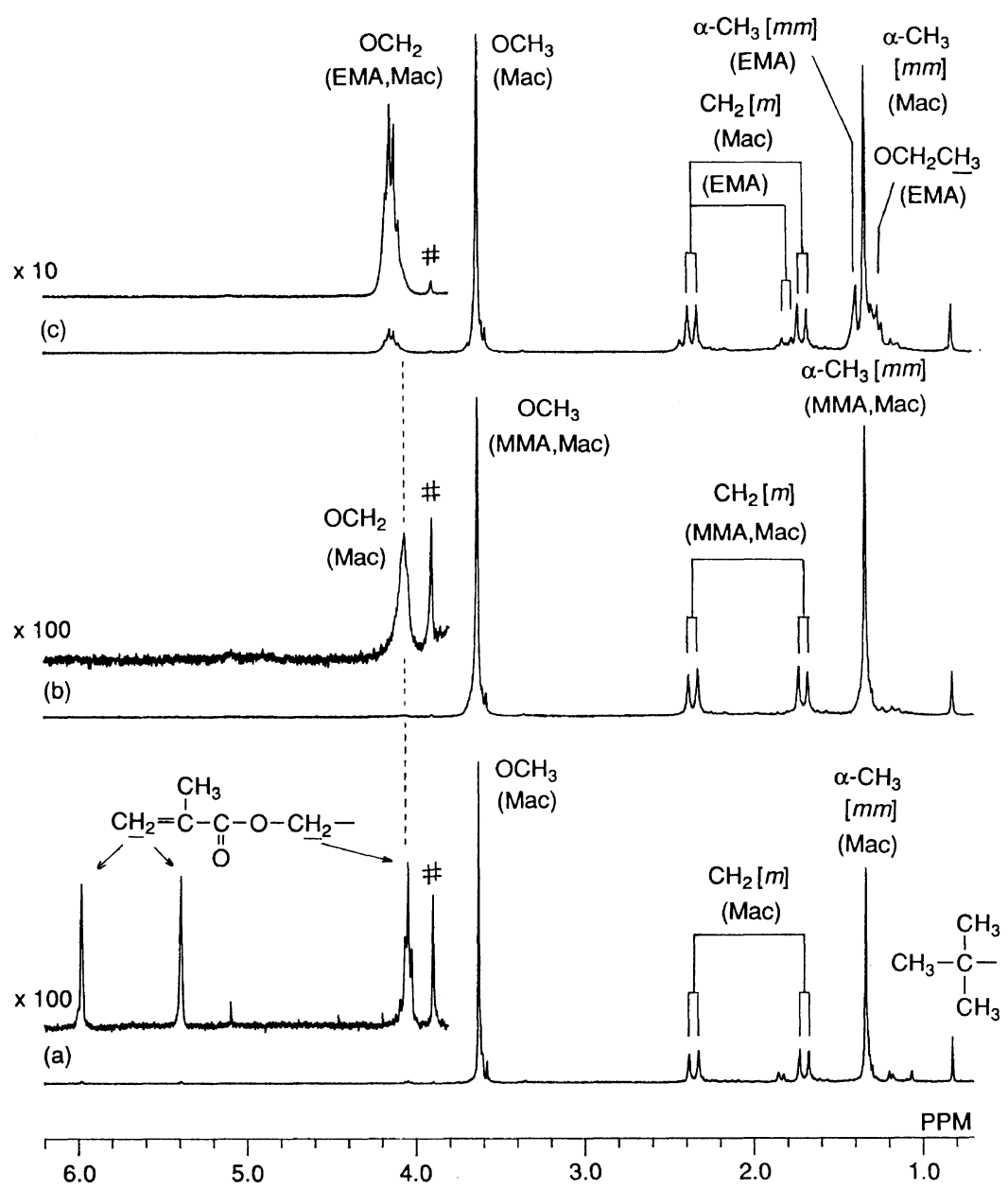

Figure 2. $270 \mathrm{MHz}^{1} \mathrm{H}$ NMR spectra measured in nitrobenzene- $d_{5}$ at $110^{\circ} \mathrm{C}$ of $i t$-PMMA macromonomer (a), and graft polymers of the it-PMMA macromonomer with MMA (b) and with EMA (c) prepared by $t-\mathrm{C}_{4} \mathrm{H}_{9} \mathrm{MgBr}$ in toluene at $-78^{\circ} \mathrm{C} .{ }^{13} \mathrm{C}$ satellite signal of $\mathrm{OCH}_{3}$ signal. 


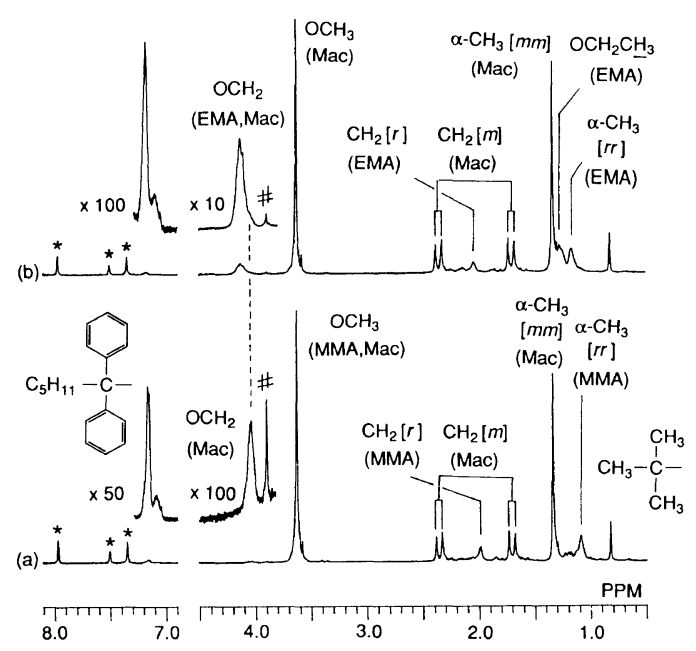

Figure 3. $270 \mathrm{MHz}{ }^{1} \mathrm{H}$ NMR spectra measured in nitrobenzene- $d_{5}$ at $110^{\circ} \mathrm{C}$ of graft polymers of $i t$-PMMA macromonomer with MMA (a) and with EMA (b) prepared by DPHLi in THF at $-78^{\circ} \mathrm{C}$. ${ }^{13} \mathrm{C}$ satellite signal of $\mathrm{OCH}_{3}$ signal. * Signals due to the remaining protons in nitrobenzene- $d_{5}$.

the macromonomer units, and the signal of $\mathrm{CH}_{3}$ protons of the ester group of EMA overlaps with the $\alpha-\mathrm{CH}_{3}$ signals. However, the $\mathrm{CH}_{2}$ proton region is almost devoid of signals due to racemo ( $r$ ) sequence ( $c f$., Figure $3 c$ ), and the $\mathrm{CH}_{2}$ signals due to EMA units are ascribable to isotactic sequence in the main chain. Thus, both the graft polymers prepared by $t-\mathrm{C}_{4} \mathrm{H}_{9} \mathrm{MgBr}$ in toluene have isotactic main chains. These results are quite similar to those for the copolymerization of st-PMMA macromonomer by $t-\mathrm{C}_{4} \mathrm{H}_{9} \mathrm{MgBr}$ in toluene. ${ }^{12,13}$

Figure 3 shows ${ }^{1} \mathrm{H}$ NMR spectra of the graft polymers prepared with DPHLi in THF. The region of $\mathrm{CH}_{2}$ protons in these spectra showed racemo methylene $\left(\mathrm{CH}_{2}[r]\right)$ proton signals along with meso methylene $\left(\mathrm{CH}_{2}[\mathrm{~m}]\right)$ proton signals due to the it-PMMA macromonomer units, indicating that the main-chain stereoregularities of the graft polymers prepared with DPHLi in THF are syndiotactic.

Quantitative determination of triad tactici- ties*2 of the graft polymers was made from quaternary carbon signals of their ${ }^{13} \mathrm{C} N M R$ spectra. The detailed procedure for the determination of main-chain tacticity will be described later.

Determination of Composition The compositions of the graft polymers with MMA main chain prepared with $t-\mathrm{C}_{4} \mathrm{H}_{9} \mathrm{MgBr}$ and with DPHLi could be determined as 13.1 and 9.41, respectively, from the ${ }^{1} \mathrm{H}$ NMR (Figures $2 \mathrm{~b}$ and 3a) signal intensities of $\mathrm{OCH}_{2}$ (macromonomer), $\left[\mathrm{OCH}_{2}\right]$, and $\mathrm{OCH}_{3}(\mathrm{MMA}$ and macromonomer), $\left[\mathrm{OCH}_{3}\right]$, according to eq 1 ,

$$
\begin{aligned}
& \mathrm{MMA} / \text { macromonomer }= \\
& \quad\left\{\left[\mathrm{OCH}_{3}\right]-\left(1.5\left[\mathrm{OCH}_{2}\right] \times \mathrm{DP}\right)\right\} / \\
& \quad 1.5\left[\mathrm{OCH}_{2}\right]
\end{aligned}
$$

where DP (degree of polymerization) is that of the $i t$-PMMA branches which is assumed to be the same as that of the starting macromonomer. The term $\left(1.5\left[\mathrm{OCH}_{2}\right] \times \mathrm{DP}\right)$ in the numerator corresponds to the contribution of the side-chain MMA units.

The compositions of the graft polymers with EMA main chain prepared with $t-\mathrm{C}_{4} \mathrm{H}_{9} \mathrm{MgBr}$ and with DPHLi were determined as 8.90 and 7.70, respectively, from the ${ }^{1} \mathrm{H}$ NMR spectra (Figures $2 \mathrm{c}$ and $3 \mathrm{~b}$ ) by comparing signal intensities of $\mathrm{OCH}_{2}$ (EMA and macromonomer) and $\mathrm{OCH}_{3}$ (macromonomer) according to eq 2. It should be noted that $\mathrm{OCH}_{2}$ proton resonances at $4.04 \mathrm{ppm}$ are the overlap signals of the macromonomer unit ( $c f$., Figures $2 b$ or 3a) and of EMA units. The contribution of the $\mathrm{OCH}_{2}$ protons of EMA units could be estimated by subtracting the contribution of the macromonomer units $\left(\left[\mathrm{OCH}_{3}\right] / \mathrm{DP}\right)$.

$$
\begin{aligned}
& \text { EMA/macromonomer }= \\
& \quad\left\{1.5\left[\mathrm{OCH}_{2}\right]-\left(\left[\mathrm{OCH}_{3}\right] / \mathrm{DP}\right)\right\} / \\
& \quad\left(\left[\mathrm{OCH}_{3}\right] / \mathrm{DP}\right)
\end{aligned}
$$

Determination of Number of Branches In

*2 The main-chain tacticities of the graft polymers are cotacticities in a strict sense. However, the term, tacticity, is used in this paper for the simplicity. 
order to determine the number of branches per molecule $\left(N_{\mathrm{br}}\right)$, the same procedures as in the case of the graft polymer of st-PMMA macromonomer ${ }^{12,13}$ were applied. The graft polymers formed with DPHLi should contain one 1,1-diphenylhexyl group at its initiating chain end as depicted in the following structural formula (3), since DPHLi is an efficient initiator for the living polymerization of methacrylate monomers in THF at low temperature. $^{20-22}$

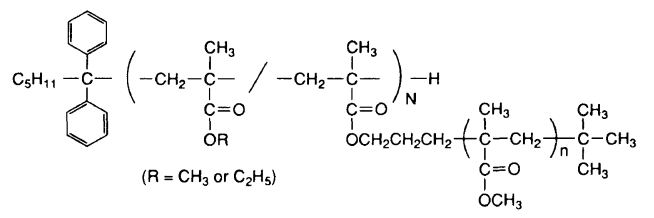

Thus, the $N_{\mathrm{br}}$ 's of the graft polymers formed with MMA and EMA could be determined as 2.89 and 3.57 , respectively, directly from the peak intensities of the phenyl protons $(6.95-7.25 \mathrm{ppm})$, [Phenyl], and $t-\mathrm{C}_{4} \mathrm{H}_{9}$ protons $(0.82 \mathrm{ppm}),\left[t-\mathrm{C}_{4} \mathrm{H}_{9}\right]$, (Figure 3 ), according to eq 4 .

$$
N_{\mathrm{br}}=\left(\left[t-\mathrm{C}_{4} \mathrm{H}_{9}\right] / 9\right) /([\text { Phenyl }] / 10)
$$

The same procedure could not be applied to the analysis of the graft polymers prepared with $t-\mathrm{C}_{4} \mathrm{H}_{9} \mathrm{MgBr}$, since the initiating chain end of the main chain and the terminal of the side chain were both $t-\mathrm{C}_{4} \mathrm{H}_{9}$ group as shown below (5), which were indistinguishable in the ${ }^{1} \mathrm{H}$ NMR spectra (Figures $2 \mathrm{~b}$ and $2 \mathrm{c}$ ).

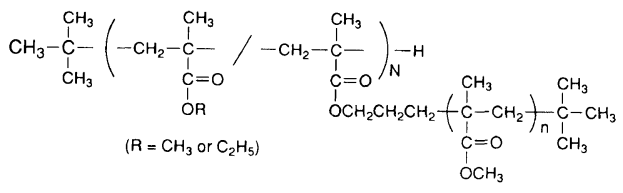

When the main chain comprises MMA units, however, the peak intensity of $t-\mathrm{C}_{4} \mathrm{H}_{9}$ at the side-chain end could be estimated from the peak intensity of $\mathrm{OCH}_{2}$ protons of the macromonomer unit ( $c f$., Figure $2 \mathrm{~b}$ ). Thus, the intensity of $t-\mathrm{C}_{4} \mathrm{H}_{9}$ protons at the initiating chain end of the main chain could be estimated by subtracting the contribution of the sidechain $t-\mathrm{C}_{4} \mathrm{H}_{9}$ group from the total $t-\mathrm{C}_{4} \mathrm{H}_{9}$ signals. The $N_{\text {br }}$ was determined as 5.47 from the signal intensities of the $t-\mathrm{C}_{4} \mathrm{H}_{9},\left[t-\mathrm{C}_{4} \mathrm{H}_{9}\right]$, and $\mathrm{OCH}_{2},\left[\mathrm{OCH}_{2}\right]$, according to eq 6 .

$$
N_{\text {br }}=4.5\left[\mathrm{OCH}_{2}\right] /\left(\left[t-\mathrm{C}_{4} \mathrm{H}_{9}\right]-4.5\left[\mathrm{OCH}_{2}\right]\right)
$$

In the case of the graft polymer with EMA main chain, the $\mathrm{OCH}_{2}$ signal of the macromonomer unit overlapped with $\mathrm{OCH}_{2}$ signal of EMA unit (Figure 2c), and thus could not be used to estimate the contribution of $t-\mathrm{C}_{4} \mathrm{H}_{9}$ group at the side-chain end. Although the contribution of $t-\mathrm{C}_{4} \mathrm{H}_{9}$ group at the side-chain end could be estimated from the intensity of $\mathrm{OCH}_{3}$ signal and the DP of the macromonomer according to eq 7, the $N_{\mathrm{br}}$ value estimated was affected strongly by even a small error in the estimation of the number of $t-\mathrm{C}_{4} \mathrm{H}_{9}$ groups of side-chain end.

$$
\begin{aligned}
& {\left[t-\mathrm{C}_{4} \mathrm{H}_{9} \text { (side chain) }\right]=} \\
& 3\left[\mathrm{OCH}_{3}\right] /(\mathrm{DP} \text { of macromonomer })
\end{aligned}
$$

Therefore, the $N_{\mathrm{br}}$ of the graft polymer was estimated from the $\bar{M}_{n}$ determined by GPC and the composition as 8.13.

Determination of $\bar{M}_{n} \quad$ From the values of the composition (C) and $N_{\text {br }}$ of the graft polymers thus determined, $\bar{M}_{n}$ 's of the graft polymers could be determined according to eq 8 , except for the case of the graft polymer prepared with EMA by $t-\mathrm{C}_{4} \mathrm{H}_{9} \mathrm{MgBr}$.

$$
\begin{aligned}
\bar{M}_{n}= & N_{\mathrm{br}} \times\left(\bar{M}_{n} \text { of macromonomer }\right) \\
& +C \times N_{\mathrm{br}} \times(M W \text { of comonomer }) \\
& +(M W \text { of initiator fragment })+1
\end{aligned}
$$

The $\bar{M}_{n}$ values estimated by GPC were slightly smaller than those determined by NMR according to eq 8 . This result may be due to the smaller hydrodynamic volume of graft polymer as compared with the corresponding linear polymer.

Quantitative Determination of Main-Chain Tacticity The $\mathrm{CH}_{2}$ proton signals in the ${ }^{1} \mathrm{H}$ NMR spectra shown in Figures 2 and 3 suggest 
qualitatively that main-chain tacticity of the graft polymer prepared with $t-\mathrm{C}_{4} \mathrm{H}_{9} \mathrm{MgBr}$ in toluene was isotactic and that prepared with DPHLi in THF was predominantly syndiotactic.

For the quantitative determination of the tacticity, ${ }^{13} \mathrm{C}$ NMR spectra of the graft polymers were measured. Figure 4 shows ${ }^{13} \mathrm{C}$ NMR spectra of quaternary carbon of graft polymers prepared from the it-PMMA macromonomer with MMA, together with that of the it-PMMA macromonomer. Figure 4 clearly indicates that the main-chain tacticity of the graft polymer prepared with DPHLi in THF (Figure 4c) differs from that of the graft polymer prepared with $t-\mathrm{C}_{4} \mathrm{H}_{9} \mathrm{MgBr}$ in toluene (Figure $4 \mathrm{~b}$ ). The triad tacticities of the whole polymer could be determined from the quaternary carbon signals as shown in Figures $4 b$ and $4 \mathrm{c}$, and should be expressed as a sum of those of the main chain and side chain.
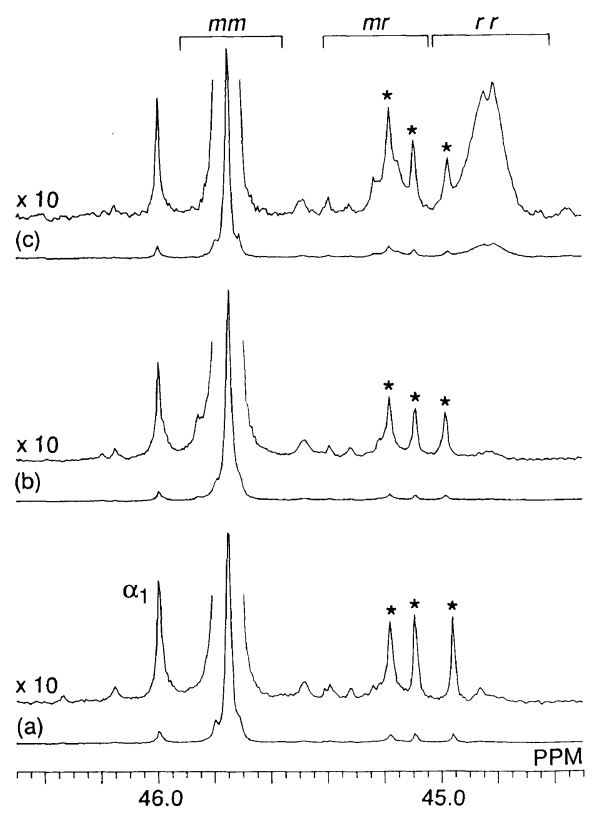

Figure 4. $67.8 \mathrm{MHz}^{13} \mathrm{C} \mathrm{NMR}$ spectra measured in chloroform- $d$ at $55^{\circ} \mathrm{C}$ of quaternary carbons of it-PMMA macromonomer (a), and graft polymers of the it-PMMA macromonomer with MMA prepared by $t-\mathrm{C}_{4} \mathrm{H}_{9} \mathrm{MgBr}$ in toluene (b) and by DPHLi in THF (c). * Signals due to quaternary carbons near the chain-ends.

$$
\begin{aligned}
& m m=x_{M} m m_{M}+x_{S} m m_{S} \\
& m r=x_{M} m r_{M}+x_{S} m r_{S} \\
& r r=x_{M} r r_{M}+x_{S} r r_{S}
\end{aligned}
$$

$m m, m r, r r$ fractions of triad tacticity obtained from the quaternary carbon.

$m m_{M}, m r_{M}, r r_{M}$; triad tacticities in the main chain.

$m m_{S}, m r_{S}, r r_{S}$; triad tacticities in the side chains.

$x_{M}, x_{S}$; mole fractions of methacrylate units in the main chain and in the side chains, respectively $\left(x_{M}+x_{S}=1\right)$.

The values of $m m_{S}, m r_{S}$, and $r r_{S}$ should correspond to those of the starting macromonomer, and $x_{M}$ and $x_{S}$ can be derived from the composition of a given graft polymer and the DP of the macromonomer. The chemical shift ranges for $m m, m r$, and $r r$ triad signals were assumed to be $45.55-45.90 \mathrm{ppm}, 45.05$ $45.40 \mathrm{ppm}$, and $44.60-45.05 \mathrm{ppm}$, respectively. Four small peaks $(44.97,45.10,45.19$, and $46.00 \mathrm{ppm}$ ) commonly observed in the three spectra are ascribable to quaternary carbons near the chain ends of the macromonomer units. From the comparison with the spectrum of it-PMMA prepared with $t-\mathrm{C}_{4} \mathrm{H}_{9} \mathrm{MgBr}$, the signal at $46.00 \mathrm{ppm}$ is assigned to the quaternary carbon in the first MMA unit at the initiating chain end $\left(\alpha_{1}\right.$ unit). ${ }^{23}$ Three other signals, which were observed in the signal regions for $m r$ and $r r$ triads overlapping with the signals of the graft polymer, were subtracted using the $\alpha_{1}$ carbon signal as an intensity standard. Based on these data, the tacticity of the main chain can be calculated using eq 9, 10, and 11 .

In the previous paper, ${ }^{12,13}$ the determination of graft polymers derived from st-PMMA macromonomer was carried out by using carbonyl carbons in the first and second MMA units from $t-\mathrm{C}_{4} \mathrm{H}_{9}$ chain end $\left(\alpha_{1} \text { and } \alpha_{2} \text { units }\right)^{23}$ of the starting st-PMMA macromonomer as an intensity standard. The corresponding signals of the it-PMMA macromonomer, however, were observed in $m m$ and $m r$ triad 
regions with complicated splitting owing to lower stereoregularity at the initiating chain end than that at in-chain unit, ${ }^{24}$ and thus not useful as an intensity standard. Therefore, the procedure using quaternary carbon signals mentioned above applied to the graft polymer derived from it-PMMA macromonomer.

The main-chain tacticities of the graft polymers with EMA main chain were also determined by the same procedure and summarized in Table $\mathrm{V}$, where some of important structural characteristics of the graft polymers are also included. The main-chain tacticities of the graft polymers prepared with $t-\mathrm{C}_{4} \mathrm{H}_{9} \mathrm{MgBr}$ in toluene and with DPHLi in THF were isotactic and syndiotactic, respectively. Stereoregularities in the MMA and EMA sequences in the main chain were close to those for PMMA and poly(EMA), respectively, formed under the same conditions; PMMA ${ }^{14}$ $m m: m r: r r=96: 4: 0$, poly(EMA) ${ }^{25} \mathrm{~mm}$ : $m r: r r=97: 3: 0 \quad\left[t-\mathrm{C}_{4} \mathrm{H}_{9} \mathrm{MgBr}\right.$, toluene $]$, PMMA $^{12} m m: m r: r r=2: 19: 79$, poly(EMA) ${ }^{12}$ $m m: m r: r r=1: 23: 76$ [DPHLi, THF]. In addition to the previous results, ${ }^{12,13}$ the present results confirm that stereospecific copolymerizations of PMMA macromonomers having methacryloyl function with methacrylate monomer are useful for the control of the main-chain stereoregularity of graft polymer.

The values of $\bar{M}_{n}$ and $N_{\mathrm{br}}$ of the graft polymers were larger than those expected from the feed ratios of the initiator and the total of the macromonomer and comonomer. These results mean that the initiator efficiencies are not $100 \%$, probably due to the difficulty of complete removal of impurities in the starting macromonomer. The present results, however, display that the anionic synthesis of the graft polymer provides us a superior way to attain structural control of the graft polymer such as tacticity and end group. Since the chain-end structures of the graft polymers are well predictable, the end-group analysis by ${ }^{1} \mathrm{H}$ NMR spectroscopy provides the detailed information on the structure of the graft polymers. For example, $N_{\mathrm{br}}$ value of the graft polymer prepared with DPHLi could be determined directly, which is more reliable than $N_{\text {br }}$ value obtained by viscometry. Moreover, $\bar{M}_{n}$ of the graft polymer could also be determined from the $N_{\mathrm{br}}$ value and the composition.

From the present and previous ${ }^{12,13}$ studies, stereoregular graft polymers with well-defined structure are now obtainable in four kinds of combination of stereoregularity in the main chain/side chains; it-/it-, it-/st-, st-/it-, and $s t-/ s t-$. These stereoregular branched polymers are useful for the investigation of the relationship between properties and stereoregularities of graft polymers.

\section{Solution Property of the Stereoregular Graft Polymer}

Solution properties of branched polymer are interesting subject by themselves. Moreover, PMMA segments of opposite stereoregularities are known to interact stereospecifically to form a stereocomplex, ${ }^{19}$ and thus solution properties of stereoregular graft PMMA are particularly interesting. Table VI summarizes the intrinsic viscosities $([\eta])$ and Huggins' coefficients $\left(k_{\mathrm{H}}\right)$ of the stereoregular graft polymers of MMA, which were obtained in the previous ${ }^{12,13}$ and present works, measured in acetone (a complex-forming solvent) and in chloroform (a non-complexing solvent) at $35^{\circ} \mathrm{C}$. The table also includes the values for linear it- and $s t$-PMMAs whose $\bar{M}_{n}$ 's are close to those of the graft polymers with it-main chain and it-side chains (it-/it-graft polymer) and with $s t$-main chain and $s t$-side chains ( $s t$ - $/ s t$-graft polymer), respectively, and the values for it -PMMA macromonomer and a mixture of it- and st-PMMAs as references.

Branched polymer generally shows lower $[\eta]$ value than linear polymer of a similar molecular weight. ${ }^{26}$ In the present case, however, the $i t$-/it-graft polymer showed $[\eta]$ values larger than those of the linear it-PMMA both in chloroform and in acetone. This is due 
to the larger weight-average molecular weight $\left(\bar{M}_{w}\right)$ of the graft polymer compared to that of the linear it-PMMA. The $\bar{M}_{w}$ values were determined by GPC-LALLS; 75400 for the graft polymer and 36900 for the linear it-PMMA. The values were used tentatively for the calculation of $[\eta]$ values based on a Mark-Houwink-Sakurada's equation for itPMMA in acetone at $30^{\circ} \mathrm{C},[\eta]=K \cdot M_{w}{ }^{a}$ $\left[K=2.3 \times 10^{-4}\left(\mathrm{dlg}^{-1}\right), a=0.63\right],{ }^{27}$ though the temperature of measurement is slightly different from the present case. The $[\eta]$ value estimated for the graft polymer is 0.272 and the observed value is 0.238 . In the case of the linear $i t$-PMMA, the $[\eta]$ value estimated from the equation is 0.173 and the observed value is 0.167 . In spite of rough estimation, the agreement between the estimated and observed $[\eta]$ values for the linear it-PMMA suggests that the slightly negative deviation of the observed $[\eta]$ value for the graft polymer from the estimated value is meaningful. This may be ascribed to the branching effect.

The same estimation of $[\eta]$ values was made for the $s t$-/st-graft polymer and the linear st-PMMA. $\bar{M}_{w}$ values determined by GPCLALLS are 41600 for the $s t$ - $/$ st-graft polymer and 30500 for the linear st-PMMA. A MarkHouwink-Sakurada's equation for atactic PMMA in acetone at $30^{\circ} \mathrm{C}$ was used for the estimation; $\left[K=0.77 \times 10^{-4}\left(\mathrm{dl} \mathrm{g}^{-1}\right), a=\right.$ $0.70] .{ }^{27}$ The results are as follows; for the $s t$-/st-graft polymer, the estimated $[\eta]$ value is 0.132 while the observed $[\eta]$ value was $0.086,{ }^{12,13}$ and for the linear st-PMMA, the estimated $[\eta]$ value is 0.106 while the observed $[\eta]$ value is $0.118 .{ }^{12,13}$ Again the agreement for the linear $s t$-PMMA between the estimated and observed $[\eta]$ values is good. The observed $[\eta]$ value for the $s t-/ s t$-graft polymer deviates negatively from the estimated $[\eta]$ value in a larger extent compared with the it-/it-graft polymer. One of the possible reasons for the result is as follows. It has been reported that $s t$-PMMA undergoes self-aggregation in certain solvents such as toluene. ${ }^{28}$ If such an aggregation of st-PMMA chains occurs in acetone, st-PMMA in acetone may exhibit a large $k_{\mathrm{H}}$ value exceeding the range usually observed. $^{26}$ In fact, both the $s t$ - $/ s t$-graft polymer and the linear st-PMMA showed $k_{\mathrm{H}}$ values exceeding unity (1.57 and 1.35 , respectively), suggesting the possibility of the self-aggregation in acetone. In the case of the $s t$ - $s t$-graft polymer, the self-aggregation takes place not only intermolecularly but also intramolecularly. Such an intramolecular aggregation is possible even at infinite dilution, and should make the graft polymer molecules compact, thus leading to the decrease of $[\eta]$ value.

In the present results, the $k_{\mathrm{H}}$ values of the $i t$ - $/$ t - and $s t$-/st-graft polymers are almost the same as those of the linear it-and st-PMMAs, respectively, both in chloroform and in acetone, while $k_{\mathrm{H}}$ values of comblike polymers (polymacromonomers) with it- and st-PMMA side chains have been reported to be larger than those of linear it- and st-PMMAs, respectively. ${ }^{8,13}$ For further discussion on the influence of branching effect on $k_{\mathrm{H}}$ values in the case of the stereoregular graft polymers, graft polymers with a wider range degree of branching should be needed and now are being prepared.

In the previous paper, ${ }^{12,13}$ we reported that a graft polymer of MMA with it-main chain and $s t$-side chains (it-/st-graft polymer) formed a stereocomplex in solution and in the solid state through intermolecular and intramolecular interactions between its it- and $s t$-PMMA chain segments. ${ }^{19}$ The intramolecular association in acetone may decrease the fraction of the chain segments available for the intermolecular association, enhancing the concentration dependence of the viscosity. In fact, the reduced viscosity of the graft polymer in acetone increased more remarkably than that of a mixture of $s t$-PMMA macromonomer and it-PMMA and that of the $s t$ - $/ s t$-graft polymer, as concentration increased, resulting in a large $k_{\mathrm{H}}$ value $(4.99) .{ }^{12,13}$ 
Table VI. Intrinsic viscosity $[\eta]$ and Huggins' coefficient $\left(k_{\mathrm{H}}\right)$ of the stereoregular graft polymers of MMA and PMMA measured in chloroform and in acetone at $35^{\circ} \mathrm{C}$

\begin{tabular}{|c|c|c|c|c|c|c|c|c|}
\hline \multirow{2}{*}{$\begin{array}{l}\text { Main } \\
\text { chain } \\
\text { (DP) }\end{array}$} & \multirow{2}{*}{$\begin{array}{l}\text { Side } \\
\text { chain } \\
\text { (DP) }\end{array}$} & \multirow{2}{*}{$N_{\text {br }}$} & \multirow{2}{*}{$\bar{M}_{n}^{\mathrm{a}}$} & \multirow{2}{*}{$\frac{\bar{M}_{w}}{\bar{M}_{n}}$} & \multicolumn{2}{|c|}{ Chloroform } & \multicolumn{2}{|c|}{ Acetone } \\
\hline & & & & & {$[\eta]$} & $k_{\mathrm{H}}$ & {$[\eta]$} & $k_{\mathrm{H}}$ \\
\hline $\begin{array}{l}i t^{-} \\
(77.1)\end{array}$ & $\begin{array}{l}i t- \\
(28.1)\end{array}$ & 5.47 & 23600 & 1.69 & 0.404 & 0.54 & 0.238 & 0.61 \\
\hline $\begin{array}{l}s t- \\
(30.1)\end{array}$ & $\begin{array}{l}i t^{-} \\
(29.5)\end{array}$ & 2.89 & 12000 & 1.30 & 0.108 & 0.83 & 0.075 & 1.36 \\
\hline $\begin{array}{c}s t^{\mathrm{b}} \\
(198.6)\end{array}$ & $\begin{array}{l}i t^{-{ }^{\mathrm{b}}} \\
(30.4)\end{array}$ & 4.02 & 32700 & 1.45 & 0.246 & 0.67 & 0.172 & 2.15 \\
\hline $\begin{array}{c}s t t^{\mathrm{c}} \\
(103.9)\end{array}$ & $\begin{array}{c}s t \mathrm{c}^{\mathrm{c}} \\
(32.1)\end{array}$ & 6.62 & 32500 & 1.35 & 0.139 & 0.86 & 0.086 & 1.57 \\
\hline $\begin{array}{l}i t^{-{ }^{c}} \\
(61.0)\end{array}$ & $\begin{array}{c}s t{ }^{-c} \\
(32.1)\end{array}$ & 2.45 & 14200 & 1.27 & 0.111 & 1.23 & 0.072 & 4.99 \\
\hline \multicolumn{3}{|c|}{$i t-\mathrm{PMMA}^{\mathrm{d}}$} & 24270 & 1.18 & 0.252 & 0.61 & 0.167 & 0.78 \\
\hline \multicolumn{2}{|c|}{$s t$-PMMA ${ }^{\mathrm{c}, \mathrm{e}}$} & & 22700 & 1.26 & 0.220 & 0.88 & 0.118 & 1.35 \\
\hline \multicolumn{3}{|c|}{ it-PMMA macromonomer $^{\mathrm{f}}$} & 3140 & 1.11 & 0.062 & 0.75 & 0.049 & 0.84 \\
\hline \multicolumn{3}{|c|}{$s t-\mathrm{PMMA}^{\mathrm{c}}+$} & & & 0.158 & 0.58 & 0.079 & 1.38 \\
\hline
\end{tabular}

${ }^{a}$ Determined by ${ }^{1} \mathrm{H}$ NMR spectroscopy. ${ }^{\mathrm{b}}$ Synthesized by the copolymerization of $i t$-PMMA macromonomer and MMA by DPHLi in THF $\left([\mathrm{MMA}]_{0} /[\mathrm{Mac}]_{0}=30\right)$. ${ }^{\mathrm{c}}$ See ref $12 .{ }^{\mathrm{d}} m m: m r: r r=97: 2: 1 .{ }^{\mathrm{e}} \bar{M}_{n}=22700 ; \bar{M}_{w} / \bar{M}_{n}=1.26$; $m m: m r: r r=0: 11: 89$. ${ }^{\mathrm{f}} m m: m r: r r=94: 3: 3$.

A similar phenomenon is expected for the graft polymer with $s t$-main chain and it-side chains (st-/it-graft polymer) obtained in this work. However, the $s t$-/it-graft polymer whose DP of the main chain was 30.1 showed smaller $k_{\mathrm{H}}$ values not only in chloroform (0.83) but also in acetone (1.36) (Table VI). The results suggest the weaker interaction between it- and st-PMMA segments in this graft polymer in acetone compared with the case of the $i t$ - $/$ st-graft polymer. In fact, a solid sample of this $s t$-/it-graft polymer recovered from acetone solution did not show an endothermic peak due to the melting of stereocomplex in DSC measurement. These results indicate that this graft polymer could not form a stereocomplex.

On the other hand, an $s t /$ it-graft polymer with a larger DP (198.6) formed a stereocomplex, which was confirmed by an endotherm at $154.7^{\circ} \mathrm{C}\left(\Delta \mathrm{H}=7.37 \mathrm{cal} \mathrm{g}^{-1}\right)$ observed in the DSC measurement of the sample recovered from acetone solution. The $k_{\mathrm{H}}$ value of this $s t$-/it-graft polymer in acetone (2.15) is significantly larger than that in chloroform $(0.67)$. The $k_{\mathrm{H}}$ value in acetone $(2.15)$ is larger than that for the mixture of $i t$-macromonomer and $s t$-PMMA (1.38), in which a stereocomplex formation may take place only intermolecularly (Table VI). These results suggest that the intramolecular complex formation results in larger $k_{\mathrm{H}}$ value, since only a part of PMMA segments can be involved in intermolecular complexation, enhancing the concentration dependence of viscosity.

The $k_{\mathrm{H}}$ value of the $s t$-/it-graft polymer in acetone (2.15) is smaller than that of the $i t$-/st-graft polymer (4.99). The fact suggests the smaller degree of intramolecular stereocomplex formation in the $s t$-/it-graft polymer. The phenomenon can be explained in the following manner. Challa and his coworkers proposed a double-stranded helix structure for PMMA stereocomplex, in which it-PMMA helical chain is surrounded by st-PMMA helix with a larger radius with the ester functions pointing outward from the double helix. ${ }^{29}$ The mod- 
el is consistent with our recent findings that a large variety of $s t$-polymethacrylates form stereocomplexes with it-PMMA and that no $i t$-polymethacrylates except it-PMMA form stereocomplex with st-PMMA. ${ }^{30-32}$ If the double-stranded helix model is adopted for the intramolecular complex formation in the st-/it-graft polymer, the main-chain st-PMMA segment should surround it-PMMA side chains. Since the main-chain mobility is restricted more than the side chain mobility, the process of such an intramolecular complex formation should be much hindered than that of the corresponding intramolecular process for the $i t$-/st-graft polymer. When the $s t$-mainchain segment is short, particularly, the complex formation becomes almost impossible as described above. The present results indicate that geometrical arrangement of it- and $s t$-PMMA segments in the graft polymers is an important factor for the intramolecular stereocomplex formation in stereoregular graft polymers.

The $i t$-/st-graft polymer whose $k_{\mathrm{H}}$ value in acetone was the largest, showed a $k_{\mathrm{H}}$ value of 1.23 in chloroform, while $k_{\mathrm{H}}$ values of the other polymers were less than unity. Therefore, the $i t$ - and st-PMMA segments in the it-/st-graft polymer may interact stereospecifically even in chloroform. This indicates that a proper geometrical arrangement of it- and st-PMMA chains in graft polymer makes stereocomplex formation possible even in the solvent formally regarded as a non-complex forming solvent.

Stereospecific interactions of stereoregular chain segments arranged in stereoregular chain architecture such as graft polymers and comblike polymers will be investigated in detail.

\section{REFERENCES}

1. R. Milkovich and M. T. Chiang, U.S. Patent, 3,786,116 (1974); R. Milkovich, Polym. Prepr., Polym. Chem. Div., Am. Chem. Soc., 21, 40 (1980).

2. P. F. Rempp and E. Franta, Adv. Polym. Sci., 58, 1 (1984).

3. Y. Yamashita, J. Appl. Polym. Sci., Appl. Polym.
Symp., 36, 193 (1981).

4. R. Asami and M. Takaki, Makromol. Chem., Rapid Commun., 12, 163 (1985).

5. K. Ute and K. Hatada, Kobunshi Kako (Polymer Application), 36, 366 (1986).

6. K. Hatada, H. Nakanishi, K. Ute, and T. Kitayama, Polym. J., 18, 581 (1986).

7. K. Hatada, T. Shinozaki, K. Ute, and T. Kitayama, Polym. Bull., 19, 231 (1988).

8. K. Hatada, T. Kitayama, K. Ute, E. Masuda, T. Shinozaki, and M. Yamamoto, Polym. Bull., 21, 165 (1989).

9. K. Hatada, T. Kitayama, E. Masuda, and M. Kamachi, Makromol. Chem., Rapid Commun., 11, 101 (1990).

10. E. Masuda, S. Kishiro, T. Kitayama, and K. Hatada, Polym. J., 23, 847 (1991)

11. T. Kitayama, S. Kishiro, and K. Hatada, Polym. Bull., 25, 161 (1991).

12. T. Kitayama, O. Nakagawa, S. Kishiro, T. Nishiura, and K. Hatada, Polym. J., 25, 707 (1993).

13. K. Hatada and T. Kitayama, "Macromolecular Design: Concept and Practice, (Macromonomers, Macroinitiators, Macroiniferters, Macroinimers, Macroiniters, and Macroinifers)," Polymer Frontiers International, Inc., New York, N.Y., 1994, pp $85-127$.

14. K. Hatada, K. Ute, K. Tanaka, Y. Okamoto, and T. Kitayama, Polym. J., 18, 1037 (1986).

15. T. Kitayama, T. Shinozaki, T. Sakamoto, M. Yamamoto, and K. Hatada, Makromol. Chem., Suppl., 15, 167 (1989).

16. H. Yuki and K. Hatada, Adv. Polym. Sci., 31, 1 (1979).

17. K. Hatada, T. Kitayama, and K. Ute, Prog. Polym. Sci., 13, 189 (1988).

18. K. Hatada, T. Kitayama, Y. Okamoto, K. Ohta, Y. Umemura, and H. Yuki, Makromol. Chem., 179, 485 (1978).

19. A. M. Liquori, G. Anzuino, V. M. Corio, M. D'Alagni, P. de Santis, and M. Savino, Nature (London), 206, 358 (1965).

20. B. C. Anderson, G. D. Andrews, P. Arthur, Jr., H. W. Jacobson, L. R. Melby, A. J. Playtis, and W. H. Sharkey, Macromolecules, 14, 1599 (1981).

21. P. Luts, P. Masson, G. Beinert, and P. Rempp, Polym. Bull., 12, 79 (1984).

22. Z-K. Cao, Y. Okamoto, and K. Hatada, Kobunshi Ronbunshu, 43, 857 (1986).

23. K. Ute, T. Nishimura, and K. Hatada, Polym. J., 21, 1027 (1989).

24. K. Hatada, K. Ute, K. Tanaka, M. Imanari, and N. Fujii, Polym. J., 19, 425 (1987).

25. T. Kitayama, K. Ute, M. Yamamoto, N. Fujimoto, and K. Hatada, Polym. J., 22, 386 (1990).

26. M. Stikler and N. Sutterlin, "Polymer Handbook," 3rd ed, J. Brandrup and E. H. Immergut, Ed., John 
Wiley \& Sons, Inc., New York, N.Y., 1989, pp VII/183.

27. M. Stikler and N. Sutterlin, "Polymer Handbook," 3rd ed, J. Brandrup and E. H. Immergut, Ed., John Wiley \& Sons, Inc., New York, N.Y., 1989, pp VII/11.

28. J. Spěváček, B. Schneider, M. Bohdanecký, and A. Sikora, J. Polym. Sci., Polym. Phys. Ed., 20, 1623 (1982).
29. E. Schomaker and G. Challa, Macromolecules, 22, 3337 (1989).

30. T. Kitayama, N. Fujimoto, Y. Terawaki, and K. Hatada, Polym. Bull., 23, 279 (1990).

31. T. Kitayama, N. Fujimoto, and K. Hatada, Polym. Bull., 26, 629 (1991).

32. K. Hatada, T. Kitayama, K. Ute, N. Fujimoto, and N. Miyatake, Macromol. Symp., 84, 113 (1994). 